\title{
Papers
}

\section{Disturbance of cerebral function in people exposed to drinking water contaminated with aluminium sulphate: retrospective study of the Camelford water incident}

\author{
Paul Altmann, John Cunningham, Usha Dhanesha, Margaret Ballard, James Thompson, \\ Frank Marsh
}

\begin{abstract}
Objective To establish whether people exposed to drinking water contaminated with 20 tonnes of aluminium sulphate in the Camelford area of Cornwall in the south west of England in July 1988 had suffered organic brain damage as opposed to psychological trauma only.

Design Retrospective study of affected people. Participants 55 affected people and 15 siblings nearest in age to one of the group but who had not been exposed to the contaminated water were studied. Main outcome measures Various clinical and psychological tests to determine medical condition and anxiety levels in affected people. Assessment of premorbid IQ (pFSIQ) with the national adult reading test, a computerised battery of psychomotor testing, and measurement of the difference in latencies between the flash and pattern visual evoked potentials in all participants.
\end{abstract}

Results The mean (SE) pFSIQ was above average at 114.4 (1.1). The most sensitive of the psychomotor tests for organic brain disease was the symbol digit coding (SDC) test (normal score 100, abnormal < 85). Participants performed less well on this test $(54.5$ (6.0)) than expected from their PFSIQ $(\mathrm{P}<0.0001)$ and a little less poorly on the averaged less discriminating tests within the battery (86.1 (2.5), $\mathrm{P}<0.0001)$. In a comparison with the 15 sibling pairs (affected people's age 41.0 (3.3) years $v$ sibling age of 42.7 (3.1) years $(\mathrm{P}=0.36)$ the exposed people had similar pFSIQ (114.7 (2.1)) to their siblings (116.3 (2.1), $(\mathrm{P}=0.59)$ but performed badly on the symbol digit coding test (51.8 (16.6)) v (87.5 (4.9) for siblings, $\mathrm{P}=0.03)$. The flash-pattern differences in exposed people were greater than in 42 unrelated control subjects of similar age (27.33 (1.64) ms $v 18.57$ (1.47) $\mathrm{ms}, \mathrm{P}=0.0002)$. The 15 unexposed siblings had significantly better flash-pattern differences than their affected siblings (13.4 (2.4) ms $v 29.6$ (2.9) ms, $\mathrm{P}=0.0002)$. No effect of anxiety could be shown on these measurements from the analysis of the anxiety scores of exposed people.

Conclusion People who were exposed to the contaminated water at Camelford suffered considerable damage to cerebral function, which was not related to anxiety. Follow up studies would be required to determine the longer term prognosis for affected individuals.

\section{Introduction}

On 6 July 1988, 20 tonnes of aluminium sulphate were accidentally emptied into the treated water reservoir that served 20000 people in the Camelford area of Cornwall. The water was heavily contaminated with aluminium and the $\mathrm{pH}$ was very low. ${ }^{1}$ Despite delay in informing the public of the accident, reports emerged of rashes and gastrointestinal disturbances within days and later musculoskeletal pains, malaise, and impairment of concentration and memory. ${ }^{1}$ Two years later about 400 people were suffering from symptoms that they attributed to the incident. The standardised hospital discharge ratios in the next 5 years were far greater than for other areas of Cornwall, ${ }^{2}$ but no systematic studies were arranged. Early reports of high blood aluminium concentrations were discounted as little effort was made to avoid sample contamination. ${ }^{3}$ One study reported psychological changes in 10 people, two of whom, who underwent bone biopsy at 6-7 months, had stainable aluminium that had disappeared at a second biopsy 1 year later. ${ }^{4}$

Three years after the incident we were asked to investigate 55 adults who were considering litigation on account of its alleged effects. We considered it unlikely that objective abnormalities could be shown because of the time interval since exposure and the difficulties in assessing a self selected group, especially with the use of subjective end points in neuropsychological tests. ${ }^{5}{ }^{6}$

We had previously investigated effects of so called safe, low level aluminium exposure in patients with end stage renal disease who were undergoing haemodialysis. In such patients the initial epidemic of aluminium related diseases was due to contamination of the water used to prepare dialysate, ${ }^{7}$ and later the importance of gastrointestinal absorption was also established. ${ }^{8-10}$ Subsequent lowering of aluminium exposure has led to the near disappearance of the florid forms of aluminium induced disease. As there was still potential

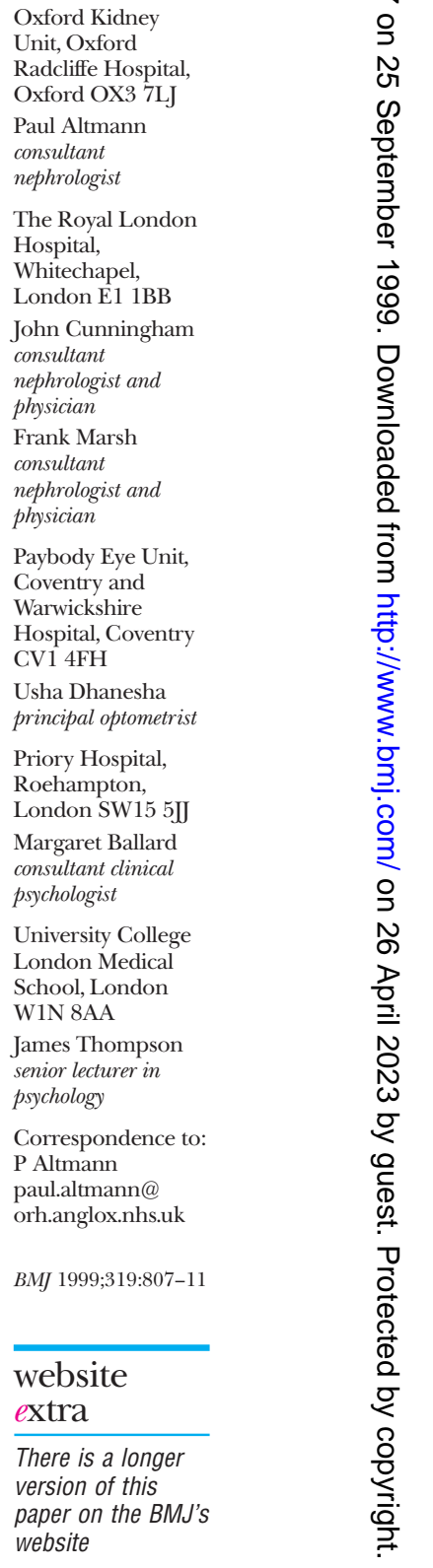

www.bmj.com 
for toxicity from modest aluminium accumulation we studied haemodialysis patients with serum concentrations averaging $55 \mu \mathrm{g} / 1$ and no clinical cerebral impairment. We found abnormalities in tetrahydrobiopterin metabolism, ${ }^{11}$ psychomotor function related to aluminium status, ${ }^{12}{ }^{13}$ and visual evoked potentials (the degree of abnormality correlating with the psychomotor defect).

In an attempt to establish whether or not the people from Camelford had suffered organic damage we undertook identical studies to the ones above. For reasons beyond our control these were performed 3 years after the incident and, as they were done in the context of litigation, could not then be published until this was resolved.

\section{Participants and methods}

We studied 55 adults who claimed to have suffered cerebral damage. All assessments were done by separate individuals without prior knowledge of the results of any of the other tests. In addition, we studied all available siblings $(n=15)$, each one nearest in age to one of the above group and who had not lived in the area of water contamination since before the incident. Standard laboratory techniques were used for routine blood haematology and biochemistry analysis. Aluminium was measured with well established methods, ${ }^{93}$ the normal serum concentration being less than $10 \mu \mathrm{g} / \mathrm{l}$. Visual analogue scales were used to score each patient's general appearance and affect and subjects' own views of their symptoms. Two identical visual analogue questionnaires were administered to examine changes in symptoms over time-one concerning symptoms 1 month after the incident (as perceived in retrospect) and one similarly relating to symptoms at the time of investigation.

\section{Psychological tests}

National adult reading test (NART)-Word reading ability correlates with IQ. It is a well established method of assessing premorbid IQ with results expressed as full scale IQ (pFSIQ) ${ }^{14}$ This was performed to establish any discrepancy between current performance and derived premorbid pFSIQ. It has a narrower range than conventional IQ testing (72-128, 100 being regarded as normal).

Bexley Maudsley automated psychological screening (BMAPS)-Psychomotor testing may be influenced by self motivated response to the set task so we used this computerised battery of tests to reduce possible interference. The second test within the battery, the symbol digit coding test, is based on the Wechsler adult intelligence scale digit symbol subtest, one of the more sensitive tests for organic brain disease. ${ }^{15}$ Symbol digit tests have been used extensively in the assessment of cognitive impairment, whatever the cause. The screening package runs on an Apple II computer. Raw data and standardised scores around a mean of 100 (calculated from normative data) are reported. Results are regarded as abnormally low if the standardised score is less than 85 (corresponding to $1 \mathrm{SD}$ below the mean) ${ }^{16}$ as in other neuropsychological tests. All the tests were carried out as outlined in the manual and our previous reports. ${ }^{12} 1316$ The symbol digit coding test is very sensitive, examining attention, motor coordination, visual scanning, and memory. ${ }^{16}{ }^{17}$ The other screening tests (numbered) are: visual spatial ability (1), visual perceptual analysis (3), verbal recognition memory (4), and visual spatial recognition memory (5). In these and previous studies, participants invariably commented that, of all the tests, the visual perceptual and spatial recognition tests were most daunting, and so we expected underperformance in these, rather than the symbol digit coding test if bias motivated by litigation was operating.

Anxiety assessment-We used the symptom checklist 90 , a multidimensional self report symptom inventory designed to measure symptomatic psychological stress, to assess anxiety. ${ }^{18}$

Visual evoked potentials

Flash and pattern stimulated visual evoked potentials were measured by standard techniques previously described. ${ }^{12}{ }^{13}$ Such measurements, carefully administered, are extremely objective and not subject to the individual's wish to underperform. After submission of summarised preliminary results to the second Clayton inquiry it had been suggested that "bias and deliberate deception" might have affected our results. ${ }^{19}$ We were careful to avoid these influences, however, making sure that the patients cooperated fully and were calm before the measurements were made.

Forty two control subjects studied separately (mean (SE) age 44 (2) years) had mean flash and pattern difference of 18.57 (1.47) ms, increasing slightly with age $(r=0.35, \mathrm{P}=0.02)$. Duplicate measurements with 50 (standard method) and 100 stimuli in a random sample of 28 exposed people were obtained, and the results were indistinguishable by paired $t$ test (50 $v 100$ flash: 120.9 (2.5) v 119.8 (2.6) ms (mean difference 1.0 $\mathrm{ms} ; 95 \%$ confidence interval -0.2 to 2.2$)$; 50 v 100 pattern: $93.6(0.9)$ v 93.5 (1.0) ms (mean difference $0.1 \mathrm{~ms}$; -1.2 to 1.4 ), confirmed by Bland and Altman comparison of methods. The method was thus reliable, and there was no added benefit from using 100 stimuli.

\section{Statistical analysis}

Results are given as means (SE) unless otherwise stated. Differences between normally distributed group data were analysed by the unpaired or paired Student's $t$ test as well as one factor analysis of variance and nonparametric tests when appropriate. Correlations between different variables were performed by least squares linear regression analysis. In addition, analysis of covariance was used when age was a covariate. Two tail probability $(\mathrm{P})$ values of $<0.05$ were regarded as significant. Analyses were performed with StatView for Macintosh. ${ }^{20}$

\section{Results}

\section{Clinical assessment}

All 55 participants (30 women and 25 men, aged 15-70 years, mean 41.8 (2.1) years) complained of short term memory loss and impaired concentration. None had any relevant personal or family history or were taking any form of psychotropic drug or consuming excessive alcohol. The participants' visual analogue scores indicated deteriorating memory and concentration (table 1). Physical examination, in particular of the 
Table 1 Summary of participants' visual analogue scores for symptoms one month after incident compared with before incident (scored -5 for major deterioration, 0 for no change, and +5 for major improvement) and at time of studies ("now") compared with before incident scored as at one month questionnaire. Negative scores bad; positive scores good

\begin{tabular}{lccc} 
Measure & One month after & Now & Change \\
\hline Memory & $-2.75(0.3)$ & $-3.42(0.2)$ & $-0.67(0.3)$ \\
\hline Concentration & $-3.05(0.2)$ & $-3.68(0.2)$ & $-0.64(0.3)$ \\
\hline Overall score & $-2.23(0.2)$ & $-2.13(0.1)$ & $0.10(0.2)$ \\
\hline
\end{tabular}

Table 2 Effects of anxiety as assessed by SCL90 on psychomotor testing (symbol digit coding test (SDC)) and visual evoked potentials

\begin{tabular}{lccc} 
Test & $\leqslant$ Median & $\geqslant$ Median & P value \\
\hline SDC test $(\mathrm{s})$ & $2.56(0.1)$ & $2.88(0.2)$ & 0.25 \\
\hline Flash-pattern difference $(\mathrm{ms})$ & $27.6(3.1)$ & $30.7(2.7)$ & 0.44 \\
\hline
\end{tabular}

neurological system and visual acuity, yielded normal results in all the participants.

\section{Psychomotor testing}

The mean pFSIQ as assessed by the national adult reading test was above average at 114.4 (1.1). All participants used English as their native language, were educated in the United Kingdom, and had no physical disability that could interfere with their ability to operate the special keyboard. Figure 1 shows the standardised results of the Bexley Maudsley screening tests. Of importance are not the absolute scores but the pattern observed. The group seemed to perform less well on the symbol digit coding test than the others, and it is this test that is thought to be most sensitive to organic brain dysfunction.

Analysis of variance confirmed that the results of the symbol digit coding test were significantly lower than any of the other results of the Bexley Maudsley screening tests $(\mathrm{P}=0.0001)$. We would not have expected the discrepancy between the patients' pFSIQ assessed by reading tests and the mean scores of Bexley Maudsley screening tests 1,3,4, and 5, even though the means of these tests fell within the normal (as stated in the manual) range (114.4 (1.1) v 86.1 (2.5), $\mathrm{P}<0.0001)$ or the even greater discrepancy relating to the symbol digit coding test (114.4 (1.1) v 54.5 (6.0), $\mathrm{P}<0.0001)$. Thus the participants performed below the level expected for their estimated premorbid IQ.

\section{Visual evoked potentials}

The mean flash-pattern difference of the Camelford participants (age 41.8 (2.1) years) was 27.33 (1.64) ms. In a group of 42 similar aged unrelated and unmatched control subjects (age 44.1 (2.3) years) the flash and pattern difference was 18.57 (1.47) ms. The difference $(8.75(2.27) \mathrm{ms})$ between the two groups was significant $(\mathrm{P}=0.0002)$. While the 2.2 year difference in age between the groups was not significant, analysis of covariance (with case and age variables entered into the model) showed that the difference in flash-pattern differences with age adjustment was a little greater at 9.45 (2.06) ms $(\mathrm{P}<0.0001)$.

As in our previous study there was a relation between the flash-pattern difference and the symbol digit coding response times $(r=0.38, \mathrm{P}=0.004)$. The association was not strong but seemed curvilinear, which is to be expected. Symbol digit coding responses and visual evoked potentials have, like all measurements of biological performance, an effective maximum speed, in that performance cannot improve infinitely. We hypothesise that participants with flash-pattern differences within the normal range will be likely to have symbol digit coding response times that lie within a normal range and there need not be any relation between the two variables observed, while as results become more abnormal the relation becomes curvilinear. There were no such relations between any of the other Bexley Maudsley screening tests and the flash-pattern difference.

\section{Effects of anxiety}

Thirty six of the 55 participants studied were available for anxiety testing, which was carried out at a different location and time. The mean SCL90 score was 1.0 (0.1), indicating relatively low levels of anxiety. Those below the median anxiety score (0.8) did not differ from those above it with regard to their symbol digit coding scores or flash-pattern differences (table 2).

\section{Sibling control study}

The 15 Camelford participants who had eligible siblings were of similar age (41.0 (3.3) years) to the whole group of 55 (41.8 (2.1) years) and to their sibling pairs (42.7 (3.1) years, mean difference $-1.7, \mathrm{P}=0.36$ ) and of similar pFSIQ as assessed by the reading test (114.7 (2.1)) to the group of 55 (114.4 (1.1)). Their siblings' results, in comparison, are shown in figure 2 together with the results of the pFSIQ, Bexley Maudsley screening tests (standardised scores), and visual evoked potential flash-pattern differences. The results show that although the sibling pairs were indistinguishable (paired Student's $t$ test) in terms of age and pFSIQ, the results of both the symbol digit coding tests and the flash-pattern differences were significantly worse (by paired Student $t$ test) in the Camelford participants than in their unexposed siblings.

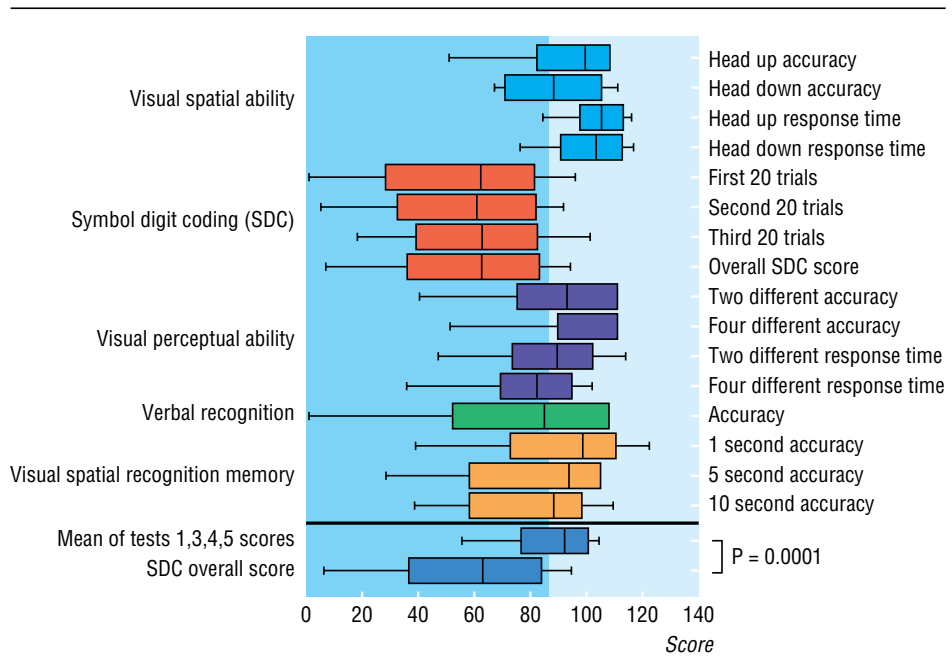

Fig 1 Summary of results of Bexley Maudsley automated psychological screening tests. In all tests results are regarded as abnormal (shaded area) if standardisation score is $<85$ (corresponding to $1 \mathrm{SD}$ ). Box plots show 10th, 25th, 50th, 75th, and 90th centiles 


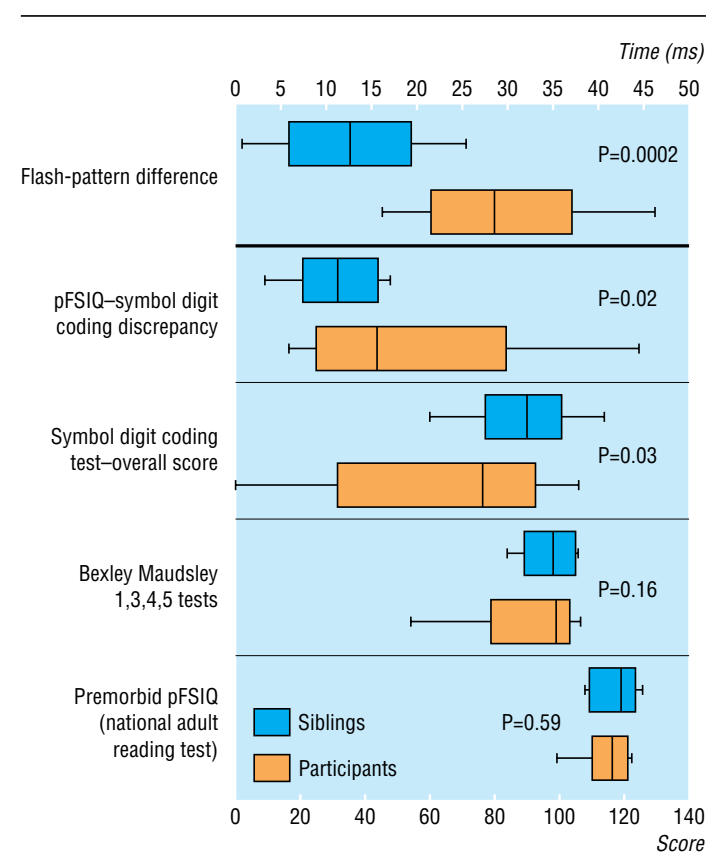

Fig 2 Sibling control studies: summary of premorbid IQ as assessed by national adult reading test ( $\mathrm{pFSIQ}$ ), scores of Bexley Maudsley automated psychological screening tests, and visual evoked potentials (top panel) in 15 participants and their siblings ( $P$ values refer to paired Student's $t$ test). Box plots as in figure 1

Haematology and biochemistry

There were no significant findings. Serum aluminium concentrations were normal, urinary aluminium concentration was increased in one participant, and several current tap water aluminium concentrations were high (containers supplied by us).

\section{Discussion}

We have shown several abnormalities in people exposed to aluminium and other contaminants in the Camelford incident. Their assessment of symptoms correlated with independent assessments of their general appearance and mental affect, adding weight to the validity of their complaints but throwing no light on the causation.

Forty two of 55 of the Camelford participants had poor psychomotor performance and all, except two, were worse on the symbol digit coding test than other tests. As a group their performance was notably worse than predicted from premorbid IQ. Visual evoked potential tests, objective measurements not influenced by subject or observer bias, were significantly different for the group when compared with the normal unrelated and unmatched controls.

The correlation between flash-pattern differences and symbol digit coding suggests that the objective disturbance in signal transmission from the eye to the optic cortex, as measured by the visual evoked potential, was associated with functional changes in psychomotor performance, in keeping with results of our previous studies, ${ }^{12}{ }^{13}$ strengthening the validity of the symbol digit coding tests in the Camelford subjects. Any suggestion that anxiety led to these abnormalities $^{321}$ is effectively rebutted by the analysis of the participants grouped according to anxiety score.
The sibling control study adds weight to the suggestion that the Camelford participants had organic brain dysfunction at the time of the study. The siblings had come from outside the area of pollution, thereby providing both out of area and genetic (phenotypic) control data. Not only were there significant differences between the siblings' symbol digit coding results and those of the Camelford participants but also quite striking differences between their flash-pattern differences (the latter measure being resistant to bias caused by litigation in favour of their affected sibling).

The pattern of abnormalities is similar to the findings we have previously described in aluminium loaded but asymptomatic patients undergoing dialysis: normal premorbid IQ with discrepant and markedly impaired symbol digit coding tests compared with other tests; and prolonged flash-pattern visual evoked potential differences. ${ }^{12}{ }^{13}$ These studies suggest the participants responded to our tests, as a group, in a manner compatible with the presence of organic brain disease and in a way similar to dialysis patients exposed to aluminium. As far as we are aware, there are no other known causes for the effects that we have described in the people from Camelford and so aluminium poisoning must be considered a possibility, although other contaminants may have contributed.

\section{Effects of aluminium exposure}

Exposure to aluminium in experimental animals, dialysis patients, patients treated with contaminated parenteral nutrition, and industrially exposed people can induce brain disease, bone disease, and anaemia. Aluminium poisoning has been reported sporadically since 1921 after acute or chronic industrial exposure, although its neurotoxicity has interested scientists since the early 19th century. Neurodegenerative changes observed in animal studies proved similar to those in Alzheimer's disease,,$^{22}$ and in 1973 Crapper et al found aluminium concentrations, approaching those in experimental models, present in certain regions of the brains of patients dying of the disease. ${ }^{23}$ Later studies supported these findings, although controversy has persisted as it has been suggested that this might be a phenomenon of ageing or an epiphenomenon. Aluminium accumulation in the neurones that contain neurofibrillary tangles ${ }^{24}$ and increased concentrations of aluminium in serum and other tissues in patients with Alzheimer's disease, ${ }^{25-27}$ however, together with some epidemiological evidence have raised concerns about the role of aluminium in this condition, ${ }^{28-30}$ although genetic factors predominate. ${ }^{31-33}$ Metabolic interactions occur between iron and aluminium, and disturbances in iron metabolism have been found in Alzheimer's disease with reduced affinity of transferrin for gallium (a chemical analogue of aluminium), possibly due to abnormally high transferrin-iron saturation. ${ }^{34}$ This could account for increased absorption of aluminium from the gut of patients with Alzheimer's disease. ${ }^{27}{ }^{35} 36$ We will never know how much aluminium was absorbed by the people in Camelford. That some aluminium was absorbed is in little doubt and, given the water $\mathrm{pH}$ and likely chemical species of aluminium salts that would have been present after the contamination, its bioavailability may well have been far greater than previously estimated. 
A review of the few psychological studies on victims of the Camelford incident concluded that the most likely explanation of the findings was that "the perception of normal and benign somatic symptoms (physical and mental) by both subjects and health professionals was heightened and subsequently attributed to an external, physical cause, such as poisoning." ${ }^{19}$ Our study suggests that this is not the case. Although there are shortcomings in the "design" of the study, for reasons beyond our control, it seems highly probable that these people had been subjected to an environmental insult that caused the effects we have reported. Through lack of funding, important wider case-control studies could not be done. It has been suggested that there are some 400 other individuals ${ }^{6}$ who have not been studied but are known to have similar symptoms, and little is known about the prognosis for affected individuals (no follow up studies have been arranged), although anecdotally many originally affected still have symptoms 11 years later. As the effects of massive aluminium contamination of water for human consumption have never been described or investigated, it is possible that this neurotoxin may have been one of the causative factors, although conclusive evidence for this is unlikely ever to be forthcoming as far as the Camelford incident is concerned.

We are grateful to Professor Sir Richard Doll, Oxford University, for his helpful comments on the manuscript; Dr Philip Day, department of chemistry, Manchester University, for his advice; David Plowman, senior biomedical scientist, department of chemical pathology, Chase Farm Hospital, Enfield, London, for the aluminium assays; and all the healthcare professionals who helped in performing these studies.

Contributors: PA (initially as senior registrar in renal and general medicine at the Royal London Hospital) designed and coordinated the studies, supervised the participants' questionnaires, performed the national adult reading and Bexley Maudsley automated screening pychomotor testing, drew the blood samples, carried out the data collation and analysis, and wrote the paper. FM participated in the instigation of the project and with JC in the study design, writing of the paper, and performance of clinical examinations. UD contributed to the study design and writing of the paper and performed all the visual evoked potential tests. MB and JT contributed to the study design and writing of the paper and performed the anxiety scoring. PA and FM are guarantors.

Funding: The studies were commissioned by lawyers acting on behalf of the plaintiffs and funded through Legal Aid.

Competing interests: None declared.

1 Clayton B, on behalf of Lowermoor Incident Health Advisory Group. Water pollution at Lowermoor, North Cornwall. Truro: Cornwall and Isles of Scilly District Health Authority, 1989.

2 Owen PJ, Miles DP. A review of hospital discharge rates in a population around Camelford in North Cornwall up to the fifth anniversary of an episode of aluminium sulphate absorption. J Public Health Med $1995 ; 17: 200-4$.

3 Clayton B, on behalf of Lowermoor Incident Health Advisory Group, Department of Health. Water pollution at Lowermoor, North Cornwall (second report). London: HMSO, 1991.

4 McMillan TM, Freemont AJ, Herxheimer A, Denton J, Taylor AP, Pazianas $\mathrm{M}$, et al. Camelford water poisoning accident: serial neuropsychological assessments and further observations on bone aluminium. Hum Exp Toxicol 1993;12:37-42.

5 Coggon D. Camelford revisited. BMJ 1991;303:1280-1.

6 Mayon-White RT. How should another Camelford be managed? BMJ 1993;307:398-9

7 Platts MM, Anastassiades E. Dialysis encephalopathy: precipitating factors and improvement in prognosis. Clin Nephrol 1981;15:223-8.

8 Kaehny WD, Hegg AP, Alfrey AC. Gastrointestinal absorption of aluminum from aluminum-containing antacids. $N$ Engl J Med 1977;296:1389-90.

9 Altmann P, Butter KC, Plowman D, Chaput de Saintonge DM, Cunningham J, Marsh FP. Residual renal function in hemodialysis patients may protect against hyperaluminemia. Kidney Int 1987;32:710-3.
Key messages

- Aluminium is a well established neurotoxin

- Accidental contamination of drinking water in Camelford by aluminium sulphate led to symptoms of loss of concentration and short term memory that were initially attributed to anxiety

- In residents exposed to the contaminated water psychomotor performance was poorer than predicted from premorbid IQ and the difference between flash-pattern visual evoked potentials was greater than normal

- Anxiety did not influence either of these measures of cerebral function

- Aluminium sulphate poisoning probably led to long term cerebral impairment in some people in Camelford

10 Priest ND, Talbot RJ, Austin JG, Day JP, King SJ, Fifield K, et al. The bioavailability of 26Al-labelled aluminium citrate and aluminium hydroxide in volunteers. Biometals 1996;9:221-8.

11 Altmann P, Al-Salihi F, Butter K, Cutler B, Blair J, Leeming R, et al. Serum aluminum levels and erythrocyte dihydropteridine reductase activity in patients on hemodialysis. N Engl J Med 1987;317:80-4.

12 Altmann P, Dhanesha U, Hamon C, Cunningham J, Blair J, Marsh F. Disturbance of cerebral function by aluminium in haemodialysis patients without overt aluminium toxicity. Lancet 1989;ii:7-12.

13 Altmann P. The toxic effects of aluminium in haemodialysis patients. London: University of London, 1991 (MD thesis).

14 Nelson HE. The national adult reading test. Windsor: NFER-Nelson, 1982

15 Lezak MD. Neuropsychological assessment. London: Oxford University Press, 1976.

16 Acker W, Acker C. Bexley Maudsley automated psychological screening and Bexley Maudsley category sorting test. Windsor: NFER-Nelson, 1982.

17 Gurling HMD, Murray RM. Investigations into the genetics of alcohol dependence and into the its effects on brain function. Third International Conference on Twin Studies, Jerusalem 1980.

18 Derogatis LR. Symptom checklist-90-R: administration, scoring and procedure manual. Baltimore: Clinical Psychometrics Research, 1977.

19 David AS, Wessely SC. The legend of Camelford: medical consequences of a water pollution accident. J Psychosom Res 1995;39:1-9.

20 Haycock KA, Roth J, Gagnon J, Finzer WF, Soper C. StatView. Berkeley, CA: Abacus Concepts, 1992.

21 McMillan TM, Dunn G, Colwill SJ. Psychological testing on schoolchildren before and after pollution of drinking water in North Cornwall. $J$ Child Psychol Psychiatry 1993;34:1449-59.

22 Terry RD, Peña C. Experimental production of neurofibrillary degeneration. 2. Electron microscopy phosphatase histochemistry and electron probe analysis. Neuropathol Exp Neurol 1965;23:200-10.

23 Crapper DR, Krishnan SS, Dalton AJ. Brain aluminum distribution in Alzheimer's disease and experimental neurofibrillary degeneration. Science 1973;180:511-3.

24 Perl DP, Brody AR. Alzheimer's disease: $\mathrm{x}$ ray spectrometric evidence of aluminum accumulation in neurofibrillary tangle-bearing neurons. Science 1980;208:297-99

25 Kellett JM, Taylor A, Oram J. Aluminosilicates and Alzheimer's disease Lancet 1986;i:682.

26 Barlow PJ, Francois PE, Goldberg I, Richardson I, Izmeth NG, Kumpeson $\mathrm{K}$, et al. Trace metal abnormalities in long-stay hyperactive mentally handicapped children and agitated senile dements. J Roy Soc Med 1986;79:581-3.

27 Mjoberg B, Hellquist E, Mallmin H, Lindh U. Aluminum, Alzheimer's disease and bone fragility. Acta Orthop Scand 1997;68:511-4.

28 Birchall JD, Chappell JS. Aluminium, chemical physiology, and Alzheimer's disease. Lancet 1988;ii:1008-10.

29 Doll R. Review: Alzheimer's disease and environmental aluminium. Age Ageing 1993;22:138-53.

30 Flaten TP, Alfrey AC, Birchall JD, Savory J, Yokel RA. Status and future concerns of clinical and environmental aluminum toxicology. J Toxicol Environ Health 1996;48:527-41.

31 Delabar J-M, Goldgaber D, Lamour Y, Nicloe A, Huret JL, de Grouchy J et al. Beta-amyloid gene duplication in Alzheimer's disease and karyotypically normal Down's syndrome. Science 1987;235:1390-2.

32 Mayeux R, Schupf N. Apolipoprotein E and Alzheimer's disease: the implications of progress in molecular medicine. Am J Public Health 1995;85:1280-4.

33 Cacabelos R. Diagnosis of Alzheimer's disease: defining genetic profiles (genotype vs phenotype). Acta Neurol Scand Suppl 1996;165:72-84

34 Farrar G, Altmann P, Welch S, Wychrij O, Ghose B, Lejeune J, et al. Defective gallium-transferrin binding in Alzheimer disease and Down syndrome: possible mechanism for accumulation of aluminium in brain Lancet 1990;335:747-50.

35 Taylor GA, Ferrier IN, McLoughlin IJ, Fairburn AF, McKeith IG, Lett D, et al. Gastrointestinal absorption of aluminium in Alzheimer's disease: response to aluminium citrate. Age Ageing 1992;21:81-90.

36 Moore PB, Edwardson JA, Ferrier IN, Taylor GA, Lett D, Tyrer SP, et al. Gastrointestinal absorption of aluminum is increased in Down's syndrome. Biol Psychiatry 1997;41:488-92.

(Accepted 9 June 1999) 